\title{
La « huitième saison » de la traduction. Quelques propos sur la traduction collective et les nouvelles pratiques traductionnelles de l'ère numérique
}

\author{
The "eighth" season of translation. \\ Comments on the collective traduction and the new \\ translation practices of the numeral era
}

\author{
Jolanta Rachwalska von Rejchwald \\ Université Marie Curie-Skłodowska, Lublin \\ jolanta.rachwalska@poczta.umcs.lublin.pl
}

\begin{abstract}
In the world 2.0, dominated by Big Data and transmedia, the figure of a translator who is alone with the complexities of the text seems to be replaced by group translations. Our era, which has become "cocreative", "co-participative", promotes collective behavior (such as coworking and crowdsourcing), also those in the field of translation. The practice of joint translations may take the form of funsubbing, which amateur translators are passionate about, but it is increasingly entering university walls (joint translations of the plays or participatory transcription of G. Flaubert's manuscripts). It is possible that the meaning to be conveyed is no longer the main driver of the activities of this new generation of collaborating translators and that there are also other goals that collaborating translators put together. This article is an attempt to describe and understand this new translation practice and its role in disseminating knowledge and culture.
\end{abstract}

Keywords: translation 2.0, collaborative/collective translation, crowdsourcing, funsubbing

\section{INTRODUCTION}

En 2011, Dieter Hornig, lors d'un colloque consacré à la traduction littéraire, constatant sa visibilité grandissante en l'espace de vingt ans, affirme que l'« ascension rapide de la traduction comme objet de réflexion n'est pas un fait unique, mais 
plutôt un phénomène périodique » (p. 26). Pour démontrer le retour cyclique de cet intérêt, il se réfère aux quatre saisons de traduction proposées par Umberto Eco auxquelles il a ajouté les trois suivantes (p. 26) ${ }^{1}$. Hilda Inderwildi, créatrice du collectif de traduction Hermaion, à l'université de Toulouse, avance une hypothèse que la traduction collective « constitue une huitième saison dans la chronologie » (2018, para. 7), proposée par Hornig. En outre, faisant remarquer le caractère intermittent de ses réapparitions, Inderwildi explique que « le traduire ensemble revient par vagues, à des époques distinctes, en fonction de situations historiques et d'objets textuels déterminés » (para. 7). Dans ce contexte, il s'impose de rappeler que la traduction collective n'est pas une invention du XXI ${ }^{\mathrm{e}}$ siècle. La traduction en équipe ou en tandem a été connue dès le $\mathrm{XII}^{\mathrm{e}}$ siècle et le $\mathrm{XX}^{\mathrm{e}}$ siècle connaît quelques emplois flamboyants de cette pratique collaborative dont la traduction de la Bible, celle d'Ulysse de Joyce ou la nouvelle traduction des œuvres de Darwin. Pourtant, la propagation de cette pratique, voire son explosion au $\mathrm{XXI}^{\mathrm{e}}$ siècle, semble intimement liée à l'impact formateur du moment socio-historique précis. En parle Henri Meschonnic qui, réfléchissant sur la transformation du traduire, nous interpelle :

Mais transformer pourquoi, comment, quand et par qui ? On ne fait pas ce qu'on veut du traduire, pas plus que de l'écrire. On se confond avec une historicité qui vous fait, quand on la fait. [...] Mais pourquoi transformer le traduire, puisque de toute manière la traduction change, ne cesse de changer ? Transformer le traduire, c'est d'abord reconnaître ce qui change, quand et pourquoi (1999, p. 119-120).

En ancrant notre raisonnement dans les dynamiques de transformation, essayons d'abord, comme nous le demande l'auteur de la Poétique du traduire, de reconnaître ce qui change et de comprendre l'impact modulateur de cette « historicité » qui est la nôtre. Il faut se rendre à l'évidence que cette " huitième saison » de traduction dans l'histoire humaine survient dans un moment que nous appelons « révolutionnaire ». Le qualifiant de cette manière, nous faisons allusion à la rupture numérique de l'an 2000, constituant un véritable «paradigme shift» (Kuhn, 1971, p. 11) qui a fracturé la continuité du temps et notre manière de communiquer, de s'informer et de transférer les données. Sous le coup des avancées exponentielles des nouvelles technologies de l'information et de la communication (TIC), émergent des plateformes numériques telles que Facebook (2004), Twitter (2006), Instagram (2010) qui

\footnotetext{
${ }^{1}$ «Dans une conférence lors des Assises de la Traduction littéraire en Arles (1993), Umberto Eco a évoqué quatre moments forts, quatre saisons où la traduction était devenue l'objet, voire l'enjeu de réflexions importantes : dans l'Antiquité, lorsque les Romains ont commencé à traduire et à refaire les auteurs grecs; au moment où les Pères de l'Église se sont posé avec Saint-Jérôme la question de la traduction des Écritures Saintes ; quand les théologiens du Moyen Âge se sont aperçus qu'ils pouvaient connaître la pensée ancienne à travers des traductions de l'arabe d'abord, et du grec ensuite (l'École de Tolède) ; et enfin entre l'humanisme (ce retour ad fontes) et la Réforme protestante avec la traduction de la Bible en langue vernaculaire » (Hornig, p. 26).
} 
consacrent l'avènement de «l'ère télématique du Web participatif » (Inderwildi, para. 7). Ces nouvelles agoras de la modernité favorisent l'échange, débloquent l'énergie de la foule, libérant son intelligence collective (crowdsourcing), ce qui induit la déhiérarchisation des rapports auteur-récepteur sensu largo ainsi que le transfert réticulaire des idées.

L'époque du web 2.0, dit collaboratif, a été prise d'assaut par le préfixe $c o-$, devenant co-créative, co-participative, vouée au coworking, au crowdsourcing, aux convergences (Jenkins, 2006) et aux conversciences. Cette vertigineuse évolution du web social a induit de profondes mutations sociales, en transformant les internautes en «webacteurs»: «ceux qui se contentaient autrefois d'une simple recherche, y cherchent des possibilités d'agir, de s'exprimer» (Goncharova \& Lacour, 2011, para. 1).

Étant donné que les formes et modalités de la traduction évoluent au rythme des transformations de la société numérique 2.0, voire de celui 3.0- dominée par les Big Data et l'univers du transmédia - l'image traditionnelle du traducteur, créateur génial, mais solitaire, "un ermite dur au travail, un marginal, un imposteur plus qu'un médiateur » (Gambier, 2016, para. 6), censé s'affronter, laborieusement et tout seul, aux aspérités du sens, devient obsolète et se voit de plus en plus concurrencée par les nouvelles pratiques de traduction. Et puisqu'il est impossible de séparer la pratique du traduire du contexte social, de l'historicité qui la fait émerger, cette nouvelle pratique traductionnelle tendra, tout comme la société 2.0 , vers une forme collective, participative et collaborative, étant donné que : «[...] les stratégies de traduction reflètent la fluctuation des littératures [et des mentalités] au long des siècles. Les temps changent, les critères moraux, esthétiques aussi... »(Lungu-Badea, 2010, p. 37).

La méthodologie de notre étude s'appuie sur la description des pratiques de traduction collaborative, réalisées par des universitaires français, et se propose un double objectif. Dans un premier temps, notre attention sera focalisée sur le mode opératoire adopté par des projets collectifs/collaboratifs, afin de comprendre comment se partagent les rôles au sein des collectifs de traduction, quelles sont les relations qui se nouent entre tous les acteurs du processus de traduction, comment se gèrent les individualités au sein du travail collectif/collaboratif ; dans un deuxième temps, nous chercherons à expliciter les rapports qui s'établissent au sein de la traduction collaborative entre les contributeurs et le texte-cible. La partie conclusive contiendra des éléments de réponse sur la portée et les enjeux de cette pratique traductionnelle.

Le point de départ de notre argumentation prend son assise dans la tension qui s'instaure entre le traduire solitaire et le traduire collectif, entre une approche individualiste et celle collaborative (Gambier, 2016, para. 16). Certains chercheurs continuent à faire perpétuer le topos de génialité du traducteur qui exerce « un grand métier de solitaire », qui est attelé à une tâche qui est « un acte de création, exigeant une fuite hors du monde » et persistent à voir dans son « isolement un corrélat de la 
qualité de [son] travail » (Kalinowski, 2002, p. 48). Mais il y a d'autres qui dénoncent « un tabou qui traverse l'histoire de la traduction » sous la forme du "paradigme romantique du créateur comme génie solitaire » (Peeters, 2015). Yves Gambier va encore plus loin, y voyant beaucoup plus que la persistance d'un paradigme suranné : "Les traductions collectives remettent en cause une certaine idéologie qui assure qu'une traduction est toujours un acte individuel centré sur un texte écrit » (2016, para. 15). Pourtant, cette posture romantique du traducteur se voit redéfinie, à l'ère du numérique, en termes d'« ingénieur en communication multilingue et multimédia » (Gouadec, 2002), en termes qui font ressortir la technicité de sa tâche. La tension repérable entre ces deux conceptions et statuts est liée au problème fondamental de l'identité de l'instance traduisante, et de sa place au sein de l'acte du traduire dont parle Henri Meschonnic :

On peut voir l'histoire de la traduction, et ses transformations en cours, comme une histoire du sujet. L'histoire des transformations du traduire, comme celle des formes littéraires, serait un aspect des formes de l'individuation [...] (p. 197).

Dans une synthèse, embrassant les années 1950-1999, Teresa Tomaszkiewicz parle de différentes mutations observables dans les pratiques de traduction au cours de ce temps et dans la manière d'appréhender le rôle du traducteur dans le processus de traduction. En aboutissant à la conclusion similaire que Meschonnic, elle fait remarquer un tournant d'individuation, centré sur la personne du traducteur qui, d'un agent invisible du processus du traduire, transférant un contenu entre deux langues, gagne en visibilité et passe au rôle du traducteur-sujet, considéré comme un acteur principal de l'acte de communication, avec tout ce que ce statut identitaire requiert en potentiel non seulement cognitif, mais aussi sensible. Cette évolution s'oriente donc du traducteur « invisible» au traducteur «visible», sujet du processus (Tomaszkiewicz, 2016, p. 49). Eu égard à l'évolution du web social 2.0, de laquelle nous avons pris un élan argumentatif, et en prenant en considération la dynamique propre à tout processus, il y a lieu de constater que cette recherche de "visibilité » ou un désir d' « individuation », évoqués par les chercheurs précités, prend, à l'heure du numérique une forme particulièrement exacerbée. On pourrait même avancer une hypothèse que la prolifération des pratiques traductionnelles marque un collaborative turn dans l'évolution de la traduction et participe dans la redéfinition du statut identitaire du traducteur.

Pour parler avec efficacité de la traduction collaborative, il s'impose de noter un flagrant flou terminologique qui interpelle tout un chacun qui s'apprête à explorer la traduction $2.0:$ on l'appelle soit collective, soit collaborative, soit participative ; on parle aussi de traduction à quatre mains ou de traductions plurielles. L'interchangeabilité de ces termes prouve que le phénomène reste encore mouvant. D'ailleurs, cette sensation de flou n'a rien de subjectif et n'a pas été seulement la nôtre, car 
Bellomo et Kaufman, co-auteurs de l'article qui demeure l'une des premières tentatives théoriques de stabiliser l'outillage notionnel et de circonscrire le champ de traduction collective en France, ont constaté qu'il s'agit « d'un objet théorique peu, voire très peu étudié par la recherche universitaire » (2015, para. 1). Ayant constaté ce « flou » terminologique (para. 5), ils sont aussi les seuls, à notre connaissance, à avoir établi, d'une manière raisonnée et méthodique, la distinction entre la traduction collective et celle collaborative.

P. Bellomo et N.N. Kaufman, dans un article ayant pour ambition de poser les bases de la pensée théorique de la traduction collective, font la distinction entre la traduction collective et collaborative :

Ce qui est ici nommé traduire collectif est un traduire qui, dans les expériences qui nous occupent, s'est accompli dans un espace et dans un temps commun, en présentiel. Cette unité de lieu et de temps a permis une démultiplication des possibles traductions d'un texte, fruit à la fois des propositions individuelles et de la circulation de ces mêmes propositions, mouvement d'échange qui en suscite d'autres jusqu'à la sensation d'avoir fait le tour des possibles. Cette démultiplication nous semble être le propre du traduire collectif. [...] Les autres pratiques, relevant d'un travail de traduction et révisions successives et souvent solitaires, relèvent du traduire collaboratif (para. 5-6).

À se tenir à cette distinction, la plupart des projets qui seront abordés dans cette étude seront mixes, mi-collectifs mi-collaboratifs.

Même si la pratique de la traduction collective s'avère encore un phénomène peu exploré, cependant, comme le constate Céline Frigau Manning, conceptrice et coordinatrice du collectif de traduction « La Langue du bourricot », « la question de la traduction collective connaît depuis peu un intérêt grandissant auprès des chercheurs, suscitant à l'heure du numérique d'importants chantiers de réflexion et de publication » (2018, para. 5) ; pour preuve, nous pouvons citer, les premiers, à notre connaissance, ouvrages et articles théoriques sur cette question (Cordingley \& Frigau Manning, 2016 ; Bodeux \& McKay, 2017). Céline Frigau Manning et Anthony Cordingley de Labez Arts et médiations humaines, International Association for Translation and Intercultural Studies (IATIS), Transferts critiques et dynamiques des savoirs (Paris 8), ont organisé une conférence internationale consacrée à cette thématique La traduction collaborative : de l'antiquité à Internet (5-7 juin 2014).

\section{PROJETS COLLABORATIFS NON-PROFESSIONNELS}

L'évolution du statut de traducteur professionnel s'accompagne d'une émergence de nouveaux acteurs prêts à s'investir dans l'acte de traduire. Il s'agit en fait de ces nouveaux utilisateurs, divisés par Y. Gambier en trois catégories (2016, para. 8-10), qui ne se contentent plus du rôle «subalterne » de consommateurs de traductions et qui se font adeptes de la traduction automatique ou intègrent des réseaux collectifs 
virtuels qui regroupent des traducteurs bénévoles, tels que Babels, Cucumis, Traduwiki, Wikitranslate, Google Translate, Worldwide Lexicon Project, Duolingo ou Tlhub.org., plateforme de traduction collaborative, fondée par Camille de Toledo, écrivain et plasticien, concepteur de la liste Finnegan. Ces nouveaux recrus de la traduction sont armés des technologies de l'information et de la communication (TIC), sous forme de logiciels et d'applications tels que les outils numériques d'écriture et d'annotation : cloud computing, écriveuses et de multiples outils pour la curation de contenus (Pearltrees, Scoop.it).

En outre, il y a tout une nébuleuse d'initiatives non-professionnels dans le domaine audiovisuel du sous-titrage réunissant des traducteurs-dialoguistes souvent amateurs, qui «peuvent s'associer pour des projets auxquels ils peuvent participer grâce à leurs connaissances linguistiques et culturelles » (Gambier, 2016, para. 9). Sans vouloir approfondir cette problématique qui demande une méthodologie à part, il faut évoquer des initiatives souvent spontanées, dites fansubbing, organisées par des fans des séries télévisées, des mangas ou des jeux vidéo. Même s'il s'agit des traducteurs amateurs, évoluant dans un milieu virtuel, il faut déconstruire des étiquettes souvent simplistes pour apprendre à reconsidérer le travail des fansubbers qui est, comme l'explique M. Boudraa, « un travail collaboratif, qui s'effectue en équipes bien rôdées et qui demande un investissement temporel et personnel ainsi que des compétences intellectuelles et techniques non négligeables » (2014). Leur travail collaboratif de traduction, bien que spontané, est bien organisé, des traducteurs étant relayés par des relecteurs et les éditeurs qui veillent sur la pertinence de la traduction et la correction de langue. Ce qui a été mis en avant par des nombreux chercheurs, dont L. Allard (2005, p. 145-172) ou E. Dagival et L. Tessier (2006, p. 117-130), c'est que le fansubbing, en tant qu'un « acte de médiation culturelle » s'inscrit dans la «culture de la convergence » (Jenkins, 2006) et « tire sa force de l'intelligence collective, puisque cette activité est un travail collectif et collaboratif qui met en avant des compétences techniques et intellectuelles » (Boudraa, 2014) de ces jeunes amateurs-bénévoles. Ce qu'il faut mettre en avant dans le cas des traductions collectives non-professionnelles, c'est qu'il s'agit d'un travail non rémunéré et volontaire, exécuté souvent par des anonymes qui se réunissent spontanément autour d'un projet à accomplir, qui les intéresse. Étant donné que les traductions dans lesquelles ils s'engagent sont ponctuelles, le texte-cible est envisagé en termes de « pro-jet», limité dans le temps, de rendu et non pas en termes d'un « labeur de création » ou d'un « accomplissement d'une œuvre » (Kalinowski, 2002, p. 48). Par conséquent, le texte-cible fait taire l'originalité des collaborateurs, gomme les différences, tendant à neutraliser sa filiation plurielle. Pourtant, la gratuité de leur action fait réfléchir; celui qui voulait chercher leurs motivations, il pourrait les trouver, d'une part, dans l'hypothétique « pulsion de traduire » (Berman, 1990, p. 6) mais, de l'autre, dans le besoin individuel de reconnaissance, peut-être même, dans celui de l'individuation, propre au traducteur professionnel. 
Dans cette brève présentation des pratiques de traduction collective, nous passons sous silence les réseaux de traducteurs activistes, travaillant, sous la bannière des organisations non-gouvernementales, pour des causes sociales ou humanitaires (Gambier, 2007), ainsi que tous les cas des «pseudo-collaborations », signes d'une « ubérisation de la traduction » (Leclerq, 2017, para. 1) dont les stratégies de travail et surtout les enjeux sont dictés par les impératifs pécuniers et par la volonté de s'assurer la meilleure rentabilité en raccourcissant le temps par un recours aux nouvelles technologies.

\section{PROJETS COLLABORATIFS UNIVERSITAIRES}

Afin de présenter quelques initiatives de traduction collaborative, nous commençons par un exemple d'une traduction pragmatique du texte multimodal qui nous permet d'entrer de plein pied dans la problématique de l'individuation des traducteurs. Une équipe composée de quatorze étudiants et de quatorze enseignants-chercheurs a travaillé sur la traduction collaborative des essais et des notices de tableaux du catalogue d'exposition 2015. «Figures de fantaisie du XVI ${ }^{\mathrm{e}}$ au XVIII ${ }^{\mathrm{e}}$ siècle», du Musée des Augustins (Hémery \& Percival, 2015). Voici un descriptif détaillé du modus operandi du projet, dirige par une chercheuse Muriel Adrien, de l'Université Jean Jaurès (Toulouse), qui fait ressortir tous les points sensibles du processus collaboratif :

À l'université, quatorze étudiants confirmés du DEMA et du CETIM (4 M2, 4 M1 et une L2) ont été rassemblés pour s'atteler à la traduction, en binôme, soit d'un essai, soit d'une série de notices. Une équipe de treize enseignants, fonctionnant en binômes également, s'est chargée de les encadrer. Une fois la répartition décidée, la procédure était la suivante : chaque étudiant du binôme faisait la traduction intégrale de son côté. Lorsque celle-ci était terminée, les deux étudiants mettaient en commun leur traduction et s'entendaient sur les meilleurs choix de formulation. Après ce travail en concertation, lorsque la traduction finale était prête, ils la transmettaient d'abord au premier encadrant, qui leur donnait ses commentaires ; puis une fois ceux-ci pris en compte et validés par l'enseignant en question, ils transmettaient la nouvelle version à l'autre enseignant, qui leur donnait ses propres commentaires, qu'ils intégraient, avant de transmettre la version finale à la coordinatrice, Muriel Adrien, qu'elle soumettait à nouveau aux encadrants pour leur feu vert avant l'envoi aux commissaires (Adrien, 2018).

Dans le retour d'expérience de ce travail effectué en binôme, deux étudiantes, Maya Coelho et Olivia Mur, ont rendu compte des exigences imposées par ce travail à la fois collectif et collaboratif. L'une des premières, la plus pertinente, mais aussi la plus paradoxale, consistait à garder l'homogénéité stylistique de la traduction : «En effet, le fait de traduire en binôme exige d'harmoniser notre production afin de donner l'illusion qu'elle a été écrite par une seule et même personne " (Coelho \& Mur, 2018, para. 5). D'une part, il s'agissait plutôt d'apprivoiser que de gommer, 
dans le texte-cible, l'hétérogénéité du groupe composé d'individualités différentes ; de l'autre, une exigence de concertation, le ping-pong verbal entre les co-traductrices leur a fait découvrir la dimension processuelle de la traduction - ouverte, infinie voire inachevable - pouvant revêtir différentes variantes stylistiques et langagières :

La traduction de groupe peut représenter un exercice difficile, permettant cependant d'observer à quel point la traduction, comme toute forme de rédaction, reste à la fois personnelle et subjective [...]. Pour ce projet précis, l'essentiel du débat a porté sur des problèmes de formulation, de stylistique. Lors de la mise en commun de nos traductions, nous avons sans cesse dû choisir entre deux propositions. Il était très intéressant d'observer comme une même phrase pouvait se décliner et produire des traductions différentes (para. 11).

En outre, les co-traductrices, ayant choix entre le mode présentiel et virtuel du travail, immergées dans l'environnement dominé par « les technologies de rupture » (Robert, 2017), optent paradoxalement, pour le mode présentiel et pour l'échange de vive voix :

C'est ainsi que nous avons pu constater que travailler ensemble et non à distance facilite énormément la tâche. Travailler en virtuel comme c'était ensuite le cas pour la relecture fait perdre plus de temps et rend le travail moins fluide. Il est parfois compliqué de s'expliquer dans un mail ou dans un commentaire de document Word, nous l'avons clairement constaté lors de ce projet (para. 11).

[...] nous nous sommes vues quotidiennement, puisqu'il nous semblait plus facile d'argumenter en faveur de nos choix de vive voix plutôt que par ordinateurs interposés (para. 7).

Finalement, leurs témoignages permettent de faire ressortir un autre point paradoxal induit par la traduction collective : celle-ci, n'étant pas consensuelle et favorisant la conflictualité constructive, constitue une opportunité pour préserver, voire cultive le dissensus, ce fondement de la pensée et le terreau de la personnalité :

Nos recherches et notre travail en binôme ont renforcé la qualité de nos propositions et nous ont permis de nous affirmer, tout en ayant ensuite l'opportunité de réaliser une relecture collaborative (para. 16).

Nous avons fortement apprécié de pouvoir dialoguer de manière si ouverte avec les enseignantes et de ce fait, nous nous sommes senties valorisées parce que nous avions le sentiment d'être considérées comme de véritables traductrices, des futures professionnelles (para. 8).

Ce qui est mis en avant dans tous les projets collaboratifs, c'est une opportunité de déhiérarchiser les rapports traditionnels entre le traducteur, auteur du texte-cible et son lecteur; c'est un moment opportun pour « une reconfiguration des rôles traditionnels du traduire [...] : il ne s'agit nullement d'abolir une hiérarchie et de 
réassigner de nouveaux rôles. L'enjeu est ailleurs, du côté de l'instabilité et du déplacement » (Bellomo \& Kaufman, 2015, para. 12). Pourtant, il faut se méfier de l'acception qu'on peut attribuer à l' « instabilité » et écouter Barthes qui a su magnifier les vertus de l'instabilité salutaire capable de rééquilibrer la dynamique des rapports interpersonnels sans les détruire :

Ce qu'il faut rendre réversible, ce ne sont pas les « rôles » sociaux (à quoi bon disputer l'« autorité » [...]), mais les régions de la parole. [...] Le problème n'est pas d'abolir la distinction des fonctions (le professeur, l'étudiant [...]), mais de protéger l'instabilité, et, si l'on peut dire, le tournis des lieux de parole (2002, p. 907).

Ce qui vaut d'être relevé, c'est que Barthes réclame non pas le « renversement » de la hiérarchie, mais juste sa «réversion » de manière à ce que la parole et la pensée ne stagnent pas, mais qu'elles puissent circuler alimentant l'échange réticulaire des créativités.

\section{COLLECTIFS DE TRADUCTION THÉÂTRALE}

Obnubilés par le caractère envahissant de la réalité numérique, nous ne pouvons pas oublier que les ambitieux projets de traduction collectives et collaboratives de fiction ne manquent pas. Il suffit de réévoquer la traduction d'Ulysse ou le projet de transcription collaborative des manuscrits du roman Madame Bovary de Gustave Flaubert, codirigé par des chercheurs de l'université de Rouen, Danielle Girard et Yvan Leclerc, considéré comme une véritable performance à l'échelle mondiale. Sur la page web du site Flaubert, mis à part des notices biographiques de cent-trente transcripteurs, il est possible de lire leurs rendus de cette expérience, ce qui demeure une lecture fort intéressante pour comprendre la popularité des projets collectifs auprès des anonymes ${ }^{2}$.

Pourtant, fidèle à la logique argumentative de ce propos, nous avons décidé de nous concentrer sur des projets collaboratifs moins connus, mais inspirants par leur originalité et innovation, nés et réalisés dans le milieu universitaire en France. Ils concernent les traductions des pièces théâtrales qui constituent un matériau textuel propice pour observer le fonctionnement du dispositif collaboratif et le rapport entre le traducteur et son texte. La difficulté majeure dans le processus de la traduction collaborative des pièces théâtrales réside dans le fait qu'elle ne doit pas seulement aboutir à un texte à lire, mais à un texte ayant la vocation d'être énoncé sur scène, afin qu'il soit « porteur d'un devenir scénique » (Déprats, 1993, p. 34), d'un geste, d'un agir. Pour faire ressortir la dimension polyphonique du texte théâtral, la traduction doit fusionner ces différentes «langues » de la théâtralité : le mot, le verbe

\footnotetext{
${ }^{2}$ Pour plus de détails, référez-vous au site Flaubert ou à l'un des articles de presse qui ont médiatisé cet événement éditorial (voir : la bibliographie).
} 
et le geste. Une telle appréhension physique et sensible du texte théâtral, qui débouche sur le texte-cible valorisant la « langue-corps » ou le « verbo-corps » (Pavis, 1990, p. 142), s'obtient par l'interposition de différents co-traducteurs et implique : " une collaboration avec un metteur ou une metteuse en scène, voire avec des comédiennes et comédiens pour une mise en bouche, mise à l'épreuve du texte traduit » (Capra \& Mazellier, 2018, para. 2).

Mis à part cette dimension physique et performative de la traduction théâtrale, qui advient au ras du texte grâce à la plurivocité des instances traduisantes, le sens du texte théâtral dépend du contexte qui le construit et le détermine, étant " chargé de tous ses non-dits, son sous-texte, ses codes théâtraux sous-jacents différents d'une culture à l'autre » (Ségol \& Serres, 2010, para. 11). Or, il n'y a que la pluralité des traducteurs, disposant de compétences, d'expériences et de sensibilités différentes, qui est capable de «re-contextualiser » (Gambier, 2016, para. 13) la pièce-source afin de faire ressortir, dans la pièce-cible, non seulement sa dimension lexicale, mais surtout son précieux contexte, ce "monde implicite que recouvre le langage de l'autre » (Lederer, 1994, p. 122).

Nous évoquerons deux collectifs universitaires, Hermaion et La Langue du bourricot, dont les acquis ont été documentés dans le cadre du projet "Traduction » de l'Institut de Recherche Pluridisciplinaire Arts, Lettres et Langues (IRPALL), à l'Université de Toulouse 2-Jean Jaurès. Chacun d'eux configure différemment les axes majeurs de son traduire-ensemble.

Le collectif «La Langue du bourricot», proposé et dirigé par Céline Frigau Manning, fondé en 2012 à l'Université Paris 8, regroupait d'abord des étudiants français et italiens avant de s'ouvrir à d'autres nationalités. Mû par une volonté de faire connaître au public français des pièces du théâtre italien, il s'attache, non seulement à traduire, mais aussi à représenter une pièce par an. Puisque la traduction théâtrale est centrée sur l'aspect physique de la langue, les discussions traditionnelles «à la table » sont retravaillées au feu d'un travail avec le corps, en présence d'un metteur en scène, ce qui permet d'obtenir une véritable polyphonie des voix qui résonnent dans les registres différents de la traduction-cible qui s'élabore en commun. Frigau Manning insiste sur les vertus de la pluralité hétérogène de ce collectif, dont les membres, grâce à leurs origines géographiques, horizons intellectuels et ressourcements culturelles différents, font émerger le sens dans le va-et-vient entre la sensibilité des co-traducteurs, le texte-source et le texte-cible : «Traduire en collectif, c'est avant tout démultiplier les points de vue sur le texte» (2018, para 19).

Représentant une autre aire géographique du texte source, le collectif «Hermaion » a été créé, en 2014, par Hilda Inderwildi à l'Université de Toulouse 2, après la traduction collective de la pièce de Michel Decar Waldemarwolf. Il a été dédié à traduire et à faire publier en français des œuvres modernes de langue allemande. Son modèle organisationnel mixte, alliant l'atelier de traduction et un laboratoire de recherche, vise la traduction collective et collaborative professionnelle de la littérature. Cette démarche inédite met en interaction la pratique de la traduction 
avec la réflexion sur elle; de cette manière circulaire, le texte-cible s'interpénètre avec la théorie du traduire (Kershaw \& Nicholson, 2011). Sa conceptrice explique que le traduire collectif pour le théâtre place le traducteur dans un intarissable dialogue entre l'auteur, le metteur en scène, les comédiens, voire les spectateurs.

L'expérience de ces deux collectifs théâtraux permet de comprendre que malgré les caractéristiques de notre époque, fébrile et hectique, où le temps et l'attention sont les denrées de plus en plus rares, ces initiatives universitaires ne lésinent pas sur le temps, facteur clé dans les projets participatifs à visée mercantile. Au contraire, le temps devient leur principal allié, presque un outil dans le processus de la découverte du sens non seulement celui du texte-source, mais surtout du texte-cible, fruit de leur collaboration :

De telles pratiques, possibles dans un cadre universitaire où le temps offert par les personnes coordinatrices n'est ni compté ni monnayé - ou seulement pour une faible part supposent d'accepter un temps long, si l'on souhaite véritablement traduire ensemble, et non simplement à plusieurs (Capra \& Mazelli, 2018, para. 7).

Cette dernière opposition entre le «traduire ensemble » et le «traduire à plusieurs " prend une résonance particulière dans le contexte de la traduction collective. Il en résulte que ce n'est pas le nombre (de participants) qui décide de l'existence de la collaboration, mais les rapports qui se nouent, les échanges qui ont lieu, ce " troc » gratuit et généreux des idées entre les contributeurs, parce que le tout est beaucoup plus que la somme de ses parties et le groupe est beaucoup plus que le nombre de participants. Ce qui différencie travailler «ensemble» et travailler «à plusieurs ", ce sont justement les relations qui tissent la maille plurivocale du texte-cible. Peut-être, la relation, c'est ce qu'il y a d'essentiel pour comprendre le sens de l'effort dépensé par les anonymes dans l'acte de contribuer, pour comprendre le sens de «la passion généreuse de médiatrices et médiateurs, qui s'engagent sans compter » (Capra \& Mazelli, 2018, para. 12).

Dans les cas précités, nous avons pu observer deux attitudes envers la traduction-cible : soit on tend à uniformiser le texte-cible et à niveler les voix des contributeurs, soit on veille à faire ressortir le clash des individualités au sein de la traduction, afin de préserver le potentiel conflictuel de la différence, source de la pensée et de l'inventivité.

\section{CONCLUSION}

Vue de loin, la traduction collective, dominée par le Nombre, pourrait ressembler à la moderne Babel 2.0. Mais au lieu d'y voir le symbole de la confusion, on pourrait l'envisager comme la confluence des passions, la concordance des goûts, comme croisement, des métissages, art du vertige et de la salutaire errance » qui, faisant valoir «la multiplicité de notre monde» (Glissant, 1996, p. 45), favorise 
l'ouverture d'un espace de communication. Ce dernier terme, subissant une forte évolution sémantique dans le temps, ne signifie pas seulement une " mise en commun », un " partage », mais aussi une « transmission », un « échange » (Winkin, 2018).

Or, en paraphrasant le titre de l'article susmentionné de Teresa Tomaszkiewicz, il serait possible de parler non seulement du caractère de plus en plus trans-disciplinaire de la traduction collaborative, mais surtout de son aspect de plus en plus trans-personnel, trans-individualiste, axé sur le trans-ducere ${ }^{3}$ (Capra \& Mazellier, 2018, para. 13).

Dans cette perspective de la transduction, l'acte de traduction collective et/ou collaborative pourrait être appréhendé comme « un lieu possible de médiation entre identité du traducteur et altérité d'autres co-traducteurs » (de Carlo, 2006, para. 36-37). Le résultat de cette médiation, la traduction-cible, revêt le rôle d'un «produit commun » (Peeters, 2015) qui fait part de sa vérité multiple dès qu'on interroge les seuils et limites du travail collaboratif qui l'a fait naître.

\footnotetext{
${ }^{3} \mathrm{O}$. Bloch et W. von Wartburg suggèrent que « traduire » pourrait venir de l'italien tradurre (du latin tra ou trans // duco) : (Bloch \& Wartburg, 1996).
} 


\section{RÉFÉRENCES BIBLIOGRAPHIQUES}

Adrien, M. (2018). « Présentation du projet de traduction collective du catalogue d'exposition 2015 du Musée des Augustins ». La main de Thôt, 3, Miscellanées. La parole aux étudiants. Accessible sur le site http://revues.univ-tlse2.fr/lamaindethot/index.php?id=519.

Allard, L. (2005). « Express yourself 2.0! Blogs, podcasts, fansubbing, mashups... : de quelques agrégats interculturels à l'âge de l'expressivisme généralisée ». In É. Maigret \& É. Macé (réd.), Penser les médiacultures. Nouvelles pratiques et nouvelles approches de la représentation du monde (pp. 145-172). Paris : Armand Colin \& INA.

Barthes, R. (2002). Écrivains, intellectuels, professeurs. Euvres complètes III. Paris : Seuil.

Bellomo, P. \& Kaufman, N. (2015). « Le traduire collectif - Propositions théoriques autour d'expériences de traduction collective ». Traduire, 233, 35-48. DOI : 10.4000/traduire.742.

Berman, A. (1990). «La retraduction comme espace de la traduction ». Palimpsestes, 4, 1-7.

Bloch, O. \& Wartburg, W. von (1996). Dictionnaire étymologique de la langue française. Paris : Presses Universitaires de France.

Bodeux, E. \& McKay, C. (2015). « La collaboration en traduction : objectifs et mode d'emploi ». Traduire, 233, 68-72. DOI : 10.4000/traduire.756.

Bourdaa, M. (2015). «Le funsubbing, une pratique de médiation culturelle ». InaGlobal, la Revue des industries créatives et des médias. Accessible sur le site https://www.inaglobal.fr/nu merique/article/le-fansubbing-une-pratique-de-mediation-culturelle.

Capra, A. \& Mazellier, C. (2018). «Introduction. Traduire ensemble pour le théâtre ». La main de Thôt, 4. Accessible sur le site http://revues.univ-tlse2.fr/lamaindethot/index.php?id=632.

Carlo, M. de (2006). " Quoi traduire? Comment traduire? Pourquoi traduire? ». ELA. Études de linguistique appliquée, 141, 117-128. Accessible sur le site cairn/info/revue.

Catalogue d'exposition. Hémery A. \& Percival M. (réd.). (2015). Figures de fantaisie du XVI ${ }^{e}-X V I I I^{e}$ siècle. Toulouse : Musée des Augustin / Somogy Éditions d'Art.

Coelho, M. \& Mur, O. (2018). «Traduire pour le Musée des Augustins ». La main de Thôt, 3, Miscellanées. La parole aux étudiants. Accessible sur le site http://revues.univ-tlse2.fr/lama indethot/index.php?id=522.

Cordingley, A. \& Frigau Manning, C. (réd.). (2016). Collaborative Translation: from the Renaissance to the Digital Age. London : Bloomsbury.

Dagiral, É. \& Tessier, L. (2006). « 24 heures! Le sous-titrage amateur des nouvelles séries télévisées ». In F. Gaudez, Les arts moyens aujourd'hui. Vol. II (pp. 117-130). Paris : L'Harmattan.

Déprats, J.-M. (1993). « La spécificité de la traduction théâtrale : quelques exemples pris dans Shakespeare ». In J.-M. Déprats (réd.), Actes du Colloque du CTL - Traduire le théâtre, Je perce l'énigme, mais je garde le mystère. 16 (pp. 33-49). Lausanne : Université de Lausanne.

Frigau Manning, C. (2018). « Le gai traduire. Histoire(s) d'un collectif de traduction théâtrale à l'Université Paris 8 ». La main de Thôt, 4. Traduire ensemble pour le théâtre. Accessible sur le site http://revues.univ-tlse2.fr/lamaindethot/index.php?id=585.

Gambier, Y. (2007). « Réseaux de traducteurs/interprètes bénévoles ». Meta, 52 (4), 658-672. DOI : 10.7202/017691ar.

Gambier, Y. (2016). " Traduction et texte: vers un nouveau double paradigme ». Signata, 7, 175-197. DOI : 10.4000 /signata. 1195.

Glissant, É. (1996). Introduction à une poétique du divers. Paris : Gallimard.

Goncharova, Y. \& Latour, P. (2011). " TraduXio : nouvelle expérience en traduction littéraire ». Traduire, 225, 86-100. DOI : 10.4000/traduire.94.

Gouadec, D. (2002). Profession : traducteur. Paris : La Maison du Dictionnaire. 
Hornig, D. (2011). « Pour une anatomie de la traduction ». Accessible sur le site http://dela.univparis8.fr/etranger/pages/1-4/Hornig.pdf.

Inderwildi, H. (2018). « Le collectif HERMAION. Enjeux, méthode et idéologie ». La main de Thôt, 4. Traduire ensemble pour le théâtre. Accessible sur le site http://revues.univ-tlse2.fr/ lamaindethot/index.php?id=558.

Jenkins, H. (2006). Convergence Culture. Where old and new media collide. New York : NYU Press.

Kalinowski, I. (2002). " La vocation au travail de traduction ». Actes de la recherche en sciences sociales, 144, 47-54. DOI : 10.3406/arss.2002.2807.

Kershaw, B. \& Nicholson, M. (2011). Research Method in Theatre and Performance. Edinbourgh : Edinbourgh University Press.

Kuhn, T. (1970). La structure des révolutions scientifiques. Trad. L. Meyer. Paris : Flammarion.

Leclercq, G. (2017). « Ubérisation de la traduction : mieux la décrypter ». Traduire, 237, 5-7. DOI : $10.4000 /$ traduire.933.

Lederer, M. (1994). La traduction aujourd'hui. Le modèle interprétatif. Paris : Hachette.

Lungu-Badea, G., Pelea, A. \& Pop, M. (réd.). (2010). (En)jeux esthétiques de la traduction. Éthique(s) et pratiques traductionnelles. Timisoara : Editura Universitati de Vest.

Meschonnic, H. (1999). Poétique du traduire. Lagrasse : Verdier.

Pavis, P. (1990). Le Théâtre au croisement des cultures. Paris : Librairie J. Corti.

Peeters, B. (2015). "Écrire ensemble. Un projet inachevé ». ILCEA, 24. Accessible sur le site http://journals.openedition.org/ilcea/3523.

Robert, A.-M. (2017). « Technologies de ruptures sur le web. Les traducteurs (presque) rompus à l'exercice ». Traduire, 237, 48-56. DOI : 10.400/traduire.947.

Savigneau, J. (2006). « Madame Bovary dans tous ses états ». Le Monde des Livres. Accessible sur le site http://www.lemonde.fr/livres/article/2006/10/19/madame-bovary-dans-tous-ses-etats_8 $251713260 . h t m l$.

Ségole, M. \& Serres, K. (2010). «Écrire et traduire pour la scène : un même territoire du sensible ». Traduire, 223, 102-113. DOI : 10.4000/traduire.313.

Site Flaubert. Accessible sur le site http://flaubert.univ-rouen.fr/manuscrits/.

Tomaszkiewicz, T. (2016). « Coraz bardziej interdyscyplinarny charakter badań przekładoznawczych ». Między oryginatem a przektadem, 1 (31), 43-59.

Winkin, Y. (2018). «Communication ». Encyclopodia Universalis [En ligne]. Accessible sur le site http://www.universalis.fr/encyclopedie/communication/. 